\title{
Meander Graphs
}

\author{
Christine E. Heitsch"1 ${ }^{1}$ and Prasad Tetali ${ }^{1} \|$ \\ ${ }^{1}$ Georgia Institute of Technology, School of Mathematics, Atlanta, GA, USA
}

\begin{abstract}
We consider a Markov chain Monte Carlo approach to the uniform sampling of meanders. Combinatorially, a meander $M=[A: B]$ is formed by two noncrossing perfect matchings, above $A$ and below $B$ the same endpoints, which form a single closed loop. We prove that meanders are connected under appropriate pairs of balanced local moves, one operating on $A$ and the other on $B$. We also prove that the subset of meanders with a fixed $B$ is connected under a suitable local move operating on an appropriately defined meandric triple in $A$. We provide diameter bounds under such moves, tight up to a (worst case) factor of two. The mixing times of the Markov chains remain open.

Résumé. Nous considérons une approche de Monte Carlo par chaîne de Markov pour l'échantillonnage uniforme des méandres. Combinatoirement, un méandre $M=[A: B]$ est constitué par deux couplages (matchings) parfaits sans intersection $A$ et $B$, définis sur le même ensemble de points alignés, et qui forment une boucle fermée simple lorsqu'on dessine $A$ "vers le haut" et $B$ "vers le bas". Nous montrons que les méandres sont connectés sous l'action de paires appropriées de mouvements locaux équilibrés, l'un opérant sur $A$ et l'autre sur $B$. Nous montrons également que le sous-ensemble de méandres avec un $B$ fixe est connecté sous l'action de mouvements locaux définis sur des "triplets méandriques" de $A$. Nous fournissons des bornes sur les diamètres pour de tels mouvements, exactes à un facteur 2 près (dans le pire des cas). Les temps de mélange des chaînes de Markov demeurent une question ouverte.
\end{abstract}

Keywords: Markov chain Monte Carlo, combinatorial enumeration, noncrossing partitions, perfect matchings

\section{Introduction}

A closed meander of order $n$ is a non-self-intersecting closed curve in the plane which crosses a horizontal line at $2 n$ points, up to homeomorphisms in the plane. Meanders are easy to define and occur in a variety of mathematical settings, ranging from combinatorics to algebra, geometry, and topology to statistical physics and mathematical biology. Yet, despite this simplicity and ubiquity, how to enumerate meanders exactly is still unknown, and even sampling uniformly from this set is a tantalizing open problem.

The study of meanders is traceable back to Poincaré's work on differential geometry, and has subsequently arisen in different contexts such as the classification of 3-manifolds [KS91] and the TemperleyLieb algebra [CJ03]. Meanders can be viewed combinatorially as suitable pairs of noncrossing partitions [Sav09, Sim00]. In Section 2, we give an equivalent combinatorial interpretation of meanders as two maximally different noncrossing perfect matchings under an appropriate local move operation, motivated in part by the biological "RNA folding" problem. Since meanders are an abstraction of polymer

\footnotetext{
${ }^{\dagger}$ Partially supported by a BWF CASI.

$\ddagger$ Partially supported by the NSF grant DMS-0701043.

1365-8050 @ 2011 Discrete Mathematics and Theoretical Computer Science (DMTCS), Nancy, France
} 
folding [Lun68, Tou50], the problems of counting and sampling meanders are of interest in more applied fields as well, such as statistical physics [DF00b] and mathematical biology [Hei].

Since the early 90's, techniques from statistical physics, such as [DF00a, DFGG00, Jen00, LZ93], have provided increasingly precise conjectures about the size of $\mathcal{M}_{n}$, the set of meanders of order $n$, and the formula $\left|\mathcal{M}_{n}\right| \approx \gamma \beta^{n} n^{\alpha}$ is believed to hold asymptotically. The most successful combinatorial attack on the enumeration problem [AP05] gives the current best bounds on the exponential growth rate $\beta$, obtained using the Goulden-Jackson cluster method for an appropriate meandric language. In terms of sampling, there have been Monte-Carlo approaches [Gol00] to producing a nearly uniform meander, however bounding the bias is a challenging statistical task.

The results given here are motivated by the uniform sampling problem. If a random walk on $\mathcal{M}_{n}$ (provably) converges rapidly to its stationary distribution, then the number of meanders of order $n$ could be estimated via sampling methods [Jer03]. Hence, in Section 2, we prove that $\mathcal{M}_{n}$ is connected under suitable pairs of "balanced" local move operations on two noncrossing perfect matchings. These results yield a Markov chain Monte Carlo (MCMC) approach to sampling uniformly from $\mathcal{M}_{n}$.

The difficulty now lies in proving that the chain mixes rapidly, since analyzing this random walk is not directly amenable to the standard techniques [Jer03, MT06] of path coupling, canonical paths, or conductance. In this respect, meanders seem to resemble other combinatorial objects - such as contingency tables [DS98], Latin squares [JM96], or Eulerian tours [AK80] — where proofs of rapid mixing remain elusive. In these cases, the uniform sampling problem is regarded as hard, and local moves acting on these combinatorial objects are of interest as potential rapidly-mixing Markov chains.

Given this, in Section 3 we introduce a local move operation on the set of meanders with a fixed "bottom" $B$ below the line. The central result in Theorem 6 states that such a subset of meanders is connected under our new "meandric triple" move. Hence, we define the meander graphs $\gamma(B)$, and investigate some structural characteristics in Section 4. The structure of $\gamma(B)$ clearly depends on $B$ in some (as yet to be determined) way. Hence, these meander graphs may be of interest beyond the uniform sampling problem, since elucidating the dependencies on $B$ might shed new light on the challenging exact enumeration problem.

\section{Balancing local moves on meanders}

We begin with $\mathrm{NC}(2 n$, match $)$, the set of noncrossing perfect matchings of $2 n$ points on a line, here often referred to simply as matchings. The points are labeled in increasing index order from 1 to $2 n$, and the matching of point $i$ with $j$ for $1 \leq i<j \leq 2 n$, referred to as the arc with endpoints $i$ and $j$, will be denoted $(i, j)$. If $(i, j)$ is an arc in a (noncrossing perfect) matching, then $j-i$ is odd.

Although usually drawn above the line, we consider the single closed loop of a meander $M$ to be a particular pair of matchings, $A$ above and $B$ below. Hence, let $\mathcal{A}_{n}$ denote $\mathrm{NC}(2 n$, match $)$ with arcs above the line, respectively $\mathcal{B}_{n}$ with arcs below. Let $(A: B)$ denote the set of closed curves in the plane formed by drawing arbitrary $A \in \mathcal{A}_{n}, B \in \mathcal{B}_{n}$ on the same endpoints. In general, there are $1 \leq k \leq n$ closed loops in $(A: B)$, denoted by $c(A, B)=k$. When $c(A, B)=1$, then the single closed curve $(A: B)$ is a meander. In this case, we say that $A$ and $B$ form a meander, or are meandric, and write

$$
[A: B]=M \in \mathcal{M}_{n} .
$$

Otherwise, the $k>1$ loops of $(A: B)$ are called a system of meanders. 

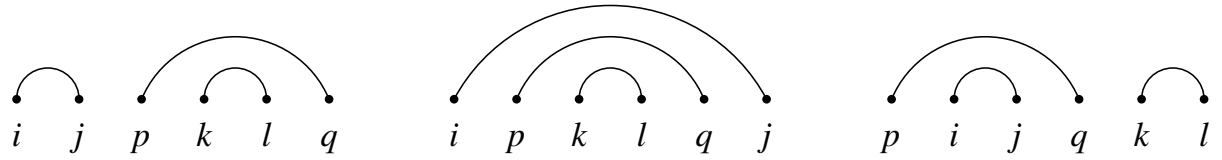

Fig. 1: The three cases where arcs $(i, j)$ and $(k, l)$ are obstructed by $(p, q)$. Only the relevant arcs are drawn.

Noncrossing perfect matchings are a combinatorial model of the biological "RNA folding" problem. As such, it is natural to consider exchanging the matching between two different arcs, which corresponds to an alternate base pairing of the corresponding RNA helices. However, this "helix exchange" operation is well-defined on matchings only when there is no obstructing arc.

Let $(i, j),(k, l) \in A \in \mathcal{A}_{n}$ with $i<k$. Since there are no crossings $i<k<j<l$, then either

$$
i<j<k<l \text { or } i<k<l<j .
$$

As illustrated in Figure 1 , the arcs $(i, j)$ and $(k, l)$ are obstructed if there is a third arc $(p, q) \in A$ with

$$
\text { either } i<j<p<k<l<q \text { or } i<p<k<l<q<j \text { or } p<i<j<q<k<l \text {. }
$$

Otherwise, they are unobstructed. Note that $(i, j)$ and $(k, l)$ are necessarily unobstructed if there exists $a \in\{i, j\}, b \in\{k, l\}$ with $|a-b|=1(\bmod 2 n)$.

Let $P=\{(i, j),(k, l)\}$ be a pair of unobstructed arcs from $A$. In this case, define a matching exchange on $P \subset A$ as the (reversible) local move operation given by

$$
\sigma_{P}(A)=\left\{\begin{array}{ll}
(A \backslash P) \cup\{(i, k),(l, j)\} & \text { if } i<k<l<j \\
(A \backslash P) \cup\{(i, l),(j, k)\} & \text { if } i<j<k<l
\end{array} .\right.
$$

The operation is not defined on obstructed arcs since a crossing would be introduced. Adopting the familial terminology from rooted trees, a matching exchange on $i<k<l<j$ converts "parent" and "child" arcs into two "siblings," and vice versa on $i<j<k<l$. Figure 2 on page 472 illustrates one of each kind. The explicit subscript $P$ may be suppressed for notational simplicity in some circumstances.

This operation is analogous to previously considered local moves on chord diagrams [MT99] and plane trees [Hei]. Via the former, it is known to give a rapidly-mixing Markov chain on $\mathrm{NC}(2 n$, match $)$. The connected graph induced on $\mathrm{NC}(2 n$, match $)$ by this operation is connected and has diameter $n-1$. Moreover, two matchings $A, B$ form a meander exactly when the geodesic path between them is diameter achieving.

More generally, the partial order on $\mathrm{NC}(2 n$, match) induced by this operation is isomorphic to the lattice of noncrossing partitions under refinement, denoted $\mathrm{NC}(n)$. Two matchings are diameter achieving if and only if their corresponding partitions are complements in the lattice. With only the exceptions from Example 1 below, each $B \in B_{n}$ has at least two distinct $A, A^{\prime} \in \mathcal{A}_{n}$, known as its Kreweras complements [Kre72], such that $[A: B],\left[A^{\prime}: B\right] \in \mathcal{M}_{n}$. Of course, there are frequently many more.

Example 1 For $1 \leq i \leq n$, let $U_{n}=\{(2 i-1,2 i)\}$ and $L_{n}=\{(1,2 n),(2 i, 2 i+1)\}$. They correspond to the top and bottom elements in $\mathrm{NC}(n)$, and hence form exactly two meanders: $\left[U_{n}: L_{n}\right]$ and $\left[L_{n}: U_{n}\right]$.

We will now define a local move operation on $M=[A: B] \in \mathcal{M}_{n}$ by operating on suitable pairs of unobstructed arcs $P \subset A, Q \subset B$ so that $\left[\sigma_{P}(A): \sigma_{Q}(B)\right]$ is again a meander. Since this is not true for arbitrary $P$ and $Q$, we first consider a matching exchange's effect on any system of meanders $(A: B)$. 


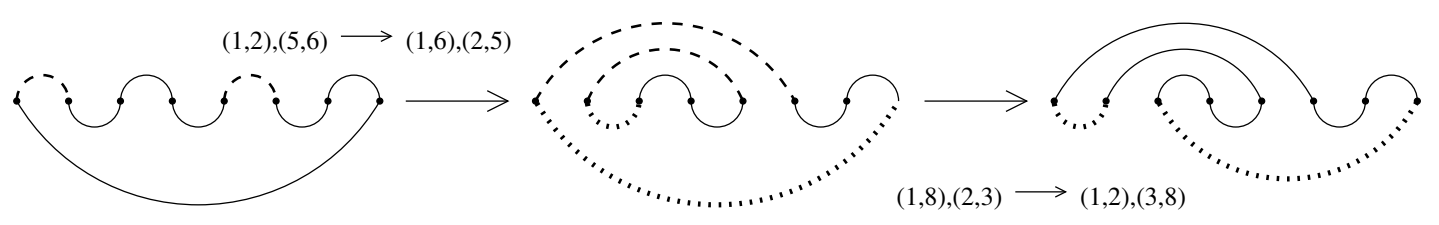

Fig. 2: Moving from the meander $\left[U_{4}: L_{4}\right]$ with the balanced matching exchanges described in Example 2

Lemma 1 Let $A \in \mathcal{A}_{n}, B \in \mathcal{B}_{n}$. Then $|c(A, B)-c(\sigma(A), B)|=1$.

Proof: Suppose $A \backslash \sigma(A)=\left\{(i, j),\left(i^{\prime}, j^{\prime}\right)\right\}$. If the unobstructed arcs $(i, j),\left(i^{\prime}, j^{\prime}\right)$ lie on the same curve from $(A: B)$, then $c(\sigma(A), B)=c(A, B)+1$. Otherwise, $c(\sigma(A), B)=c(A, B)-1$.

By symmetry, the result holds for $\sigma(B)$. Since a single exchange $\sigma(A)$ breaks the meander $[A: B]$, we show in Theorem 1 below that there always exists a compensating exchange $\sigma(B)$ which rejoins the two closed loops. Any pair of matching exchanges on $A$ and $B$ will be called balanced whenever $c(\sigma(A), \sigma(B))=1$. Thus, we can move between meanders connected by balanced pairs of local moves.

To prove this, we introduce another notation for (a system of) meanders. Recall that $j-i$ is odd for $(i, j) \in A$. If $i$ is odd, denote this arc as $i \stackrel{A}{\rightarrow} j$ and as $j \stackrel{A}{\rightarrow} i$ otherwise. Similarly, but with reversed parity, every $(2 i, 2 j-1) \in B$ is written as $2 i \stackrel{B}{\rightarrow} 2 j-1$ and $(2 j-1,2 i) \in B$ as $2 i \stackrel{B}{\rightarrow} 2 j-1$. In this way, any system of meanders is can be written as a set of ordered, alternating sequences of arcs from $A$ and $B$. Typically, we drop the $A$ and $B$ designation and simply write a meander (single closed loop) as:

$$
1 \rightarrow 2 i_{1} \rightarrow 2 j_{2}-1 \rightarrow 2 i_{2} \rightarrow \ldots \rightarrow 2 j_{n}-1 \rightarrow 2 i_{n} \rightarrow 1 .
$$

Theorem 1 Let $M=[A: B] \in \mathcal{M}_{n}$. For every pair of unobstructed arcs $P$ in $A$ there exists a pair of unobstructed arcs $Q$ in $B$ such that $c\left(\sigma_{P}(A), \sigma_{Q}(B)\right)=1$.

Proof: Suppose $i \rightarrow j$ and $i^{\prime} \rightarrow j^{\prime}$ are two unobstructed arcs from $A$. It suffices to show there exist unobstructed arcs $k \rightarrow l$ and $k^{\prime} \rightarrow l^{\prime}$ in $B$ which occur in the sequence of ordered, alternating arcs as:

$$
i \rightarrow j \ldots k \rightarrow l \ldots i^{\prime} \rightarrow j^{\prime} \ldots k^{\prime} \rightarrow l^{\prime} \ldots \rightarrow i .
$$

Let $S$ be the set of integers in $j \rightarrow \ldots \rightarrow i^{\prime}$, respectively $S^{\prime}$ in $j^{\prime} \rightarrow \ldots \rightarrow i$. Then $S$ and $S^{\prime}$ are a partition of the integers $\{1,2, \ldots, 2 n\}$ and, without loss of generality, there exists $k \in S$ and $l^{\prime} \in S^{\prime}$ such that $\left|k-l^{\prime}\right|=1(\bmod 2 n)$. Thus the $\operatorname{arcs} k \rightarrow l$ and $k^{\prime} \rightarrow l^{\prime}$ are unobstructed in $B$.

We make the previous discussion concrete by considering the following example, pictured in Figure 2.

Example 2 The meander $\left[U_{4}: L_{4}\right]$ is $1 \rightarrow 2 \rightarrow 3 \rightarrow 4 \rightarrow 5 \rightarrow 6 \rightarrow 7 \rightarrow 8 \rightarrow 1$. A matching exchange on $P=\{(1,2),(5,6)\}$ in $U_{4}$ results in two closed loops in $\left(\sigma_{P}\left(U_{4}\right): L_{4}\right): 1 \rightarrow 6 \rightarrow 7 \rightarrow 8 \rightarrow 1$ and $3 \rightarrow 4 \rightarrow 5 \rightarrow 2 \rightarrow 3$. There exist a compensating exchange on $Q=\{(1,8),(2,3)\}$ in $L_{4}$ which yields the meander $\left[\sigma_{P}\left(U_{4}\right): \sigma_{Q}\left(L_{4}\right)\right]: 1 \rightarrow 6 \rightarrow 7 \rightarrow 8 \rightarrow 3 \rightarrow 4 \rightarrow 5 \rightarrow 2 \rightarrow 1$.

The proof of Theorem 1 guarantees at least one compensating exchange $\sigma(B)$ for each $\sigma(A)$. In general, there may be many balanced pairs of exchanges for a meander $[A: B]$. For instance, there are three other matching exchanges on $B$ which would rejoin the closed loops of $\left(\sigma_{P}\left(U_{4}\right): L_{4}\right)$ in Figure 2. 
Definition 1 Let $\mathcal{G}_{n}$ be the graph whose vertices are $M, M^{\prime} \in \mathcal{M}_{n}$ and whose edges connect meanders $M=[A: B]$ and $M^{\prime}=[\sigma(A): \sigma(B)]$ with balanced pairs of matching exchanges.

Theorem 2 The graph $\mathcal{G}_{n}$ is connected.

Proof: The only meander with $\operatorname{arcs} U_{n}$ above the line is $\left[U_{n}: L_{n}\right]$. For $[A: B] \in \mathcal{M}_{n}$, there exists a sequence of matching exchanges on $A$ such that $\sigma(\ldots \sigma(A))=U_{n}$. By Theorem 1 , for each local move on the upper arcs, there is a compensating matching exchange on the bottom.

Alternatively, Theorem 2 follows from the connection between meanders and pairs of noncrossing partitions, see [Fra98, FE02] as well as [Hal06, Sav09]. In that context, the graph $\mathcal{G}_{n}$ is the Hasse diagram of the induced partial order.

Theorem 2 suggests a natural ergodic Markov chain on the state space $\mathcal{M}_{n}$ with transition probability matrix $\mathbb{P}$. We will define $\mathbb{P}\left(M, M^{\prime}\right)$ to be positive if there is a pair of balanced matching exchanges connecting $M, M^{\prime} \in \mathcal{M}_{n}$. It is also technically convenient to assume the self-loop probability is positive; $\mathbb{P}(M, M)>0$ for every $M \in \mathcal{M}_{n}$. Both sets of probabilities are specified (implicitly) below.

The fact that $\mathcal{G}_{n}$ is connected implies that such a Markov chain is ergodic; for every pair of states, there is a time by which the probability of visiting one state from the other is positive. The self-loop probability further guarantees aperiodicity; a high enough power of $\mathbb{P}$ has all entries positive, which in turn implies that the Markov chain converges to its so-called stationary distribution on $\mathcal{M}_{n}$. Finally, recall that a symmetric Markov chain has the uniform distribution as its stationary distribution. This suggests specifying the off-diagonal transition probabilities so as to make $\mathbb{P}$ symmetric.

One fairly standard way in MCMC methods of achieving a symmetric chain is to consider the so-called maximum-degree random walk. Let $\Delta\left(\mathcal{G}_{n}\right)$ be the maximum vertex degree in the meander graph $\mathcal{G}_{n}$. We have $\Delta\left(\mathcal{G}_{n}\right)=\Theta\left(n^{4}\right)$, based on a degree of $n^{2}\left(n^{2}-1\right) / 12$ for $\left[U_{n}: L_{n}\right]$ and the naive bound of $\left(\begin{array}{c}n \\ 2\end{array}\right)^{2}$ on all pairs of two arcs. Defining $\mathbb{P}\left(M, M^{\prime}\right):=1 / \Delta\left(\mathcal{G}_{n}\right)$ for every adjacent pair $M, M^{\prime}$, and $\mathbb{P}(M, M):=1-\sum_{M^{\prime} \neq M} \mathbb{P}\left(M, M^{\prime}\right)$, makes $\mathbb{P}$ symmetric and (row, hence column) stochastic.

There are several other ways to define $\mathbb{P}$ so that it is row and column stochastic, which is sufficient to guarantee uniformity of stationary probabilities. However, the seemingly challenging open question we raise here is whether the above Markov chain (or an analogous one) is "rapidly mixing" on $\mathcal{M}_{n}$ ? In other words, irrespective of the starting state at time $t=0$, is the first time the chain is within $1 / 4$ (say) in total variation distance of the uniform distribution at most polynomial in $\log \left|\mathcal{M}_{n}\right|$ ?

A second question in the same vein would be to sample uniformly from the subset of meanders with a fixed bottom $B$. Our main result (Theorem 6 below) provides, once again, a natural way to define an appropriate Markov chain which converges to the correct (uniform) distribution. However, the rate of mixing of this "meandric triple" chain also remains open.

\section{Graphing meandric triple moves}

Since the matching exchange operation gives a rapidly-mixing Markov chain [MT99] on NC(2n, match), one direction of attack on the problem of analyzing the mixing time of the Markov chain on $\mathcal{G}_{n}$ restricts to analyzing a random walk on the set of meanders $[A: B]$ with a fixed $B \in \mathcal{B}_{n}$.

We introduce such a random walk as follows. We prove that the two closed loops in $(\sigma(A): B)$ can be rejoined by a move on $\sigma(A)$ when the exchange operation is applied twice to an appropriate triple of arcs in $A$. This yields a new "meandric triple" move where $[A: B] \rightarrow[\sigma(\sigma(A)): B]$, which provably connects 
the subset of meanders with fixed $B$. Hence, we get meander graphs $\gamma(B)$ with differing structures depending on $B \in \mathcal{B}_{n}$. The study of these meander graphs may well be of interest beyond the uniform sampling problem, since elucidating the (still unknown) dependencies on $B$ might shed new light on the challenging exact enumeration problem.

Two arbitrary exchange operations on $A$ do not yield another meander since $c(\sigma(\sigma(A)), B)$ may be either 1 or 3 . Hence, we begin by defining an appropriate triple of $\operatorname{arcs}$ in $A$ on which to act.

Definition 2 Let $i \rightarrow j, k \rightarrow l$, and $q \rightarrow p$ be three arcs from $A \in \mathcal{A}_{n}$. They are a meandric triple if exactly two of the three pairs of arcs are unobstructed.

Figure 1 on page 471 illustrates the three possible configurations for a meandric triple, assuming no other obstructing arcs. Observe the equivalence of the configurations under the endpoint operations of rotation, that is $i \rightarrow i-1(\bmod 2 n)$, and reversal, that is $i \rightarrow 2 n+1-i$. We will use the fact that these operations preserve the single closed loop forming a meander in subsequent arguments.

Excepting only $U_{n}$ and $L_{n}$, any matching $A \in A_{n}$ with $n \geq 3$ contains at least one meandric triple. The maximum in any $A$, and hence the maximum degree over all meander graphs $\gamma(B)$ defined below, is $\Theta\left(n^{2}\right)$. The naive bound of $\left(\begin{array}{l}n \\ 3\end{array}\right)$ reduces to $\mathcal{O}\left(n^{2}\right)$ by observing that any meandric triple has a unique "youngest" arc. Hence, there are only $\mathcal{O}(n)$ such parent/child combinations, with an additional factor of $n$ for the third arc (either grandparent or parent's sibling). This is best possible, over all bottom arcs $B \in \mathcal{B}_{n}$, since there is a matching $A \in \mathcal{A}_{n}$ with $\lceil(n-1) / 2\rceil\lfloor(n-1) / 2\rfloor$ meandric triples.

Theorem 3 Let $M=[A: B] \in \mathcal{M}_{n}$. There exists a sequence of two matching exchange operations on a meandric triple in A such that $c(\sigma(\sigma(A)), B)=1$.

Proof: Let $i \rightarrow j, k \rightarrow l$, and $q \rightarrow p$ be a meandric triple from $A$ where $i \rightarrow j$ and $q \rightarrow p$ are unobstructed, $q \rightarrow p$ and $k \rightarrow l$ are unobstructed, and

$$
i \rightarrow j \overbrace{\cdots}^{R} q \rightarrow p \underbrace{\ldots}_{S} k \rightarrow l \overbrace{\cdots}^{T} .
$$

The six different cases for the ordering of $i, j, \ldots$ along the horizontal line are equivalent under rotation and reversal. Hence, suppose that

$$
i<p<k<l<q<j .
$$

By assumption, the exchange $\sigma_{P}(A)$ is defined for $P=\{(p, q),(k, l)\}$. Furthermore, $(p, k),(l, q)$ and $(i, j)$ are all unobstructed in $\sigma_{P}(A)$. The two closed loops of $\sigma_{P}(A)$ are rejoined into the meander

$$
i \rightarrow p \underbrace{\ldots}_{S} k \rightarrow j \overbrace{\cdots}^{R} q \rightarrow l \overbrace{\cdots}^{T}
$$

by operating on $Q=\{(i, j),(p, k)\}$ which results in $(i, p),(k, j) \in \sigma_{Q}\left(\sigma_{P}(A)\right)$.

Definition 3 Let $i \rightarrow j, k \rightarrow l$, and $q \rightarrow p$ be a meandric triple in $A$ for $M=[A: B] \in \mathcal{M}_{n}$ with $i \rightarrow j \ldots q \rightarrow p \ldots k \rightarrow l \ldots$ Define a meandric move on $M$, denoted $\tau(M)=[\sigma(\sigma(A)): B]$, as the pair of matching exchanges which replaces the meandric triple in $A$ with $i \rightarrow p, k \rightarrow j$, and $q \rightarrow l$. 

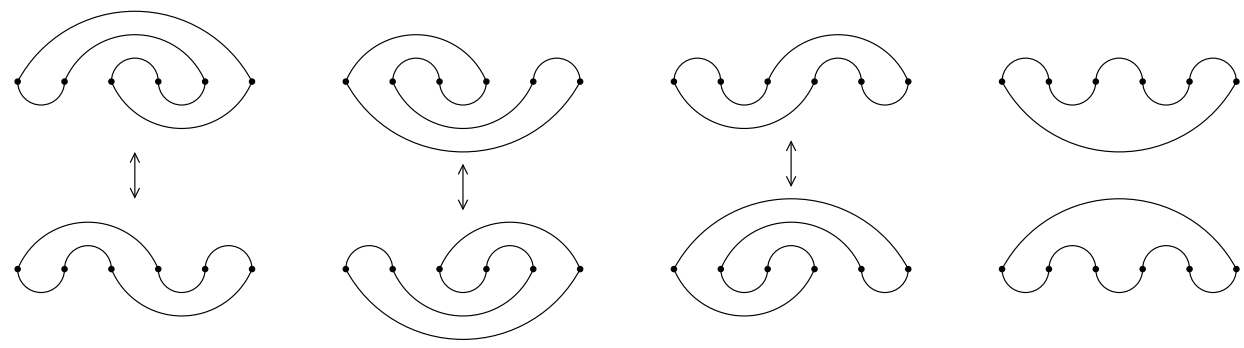

Fig. 3: The eight meanders from $\mathcal{M}_{3}$ with the three connections under a meandric move.

Figure 3 illustrates that, for a given meandric triple in $A$, there is exactly one way of preserving a single closed loop while exchanging the matchings among the six endpoints. Also note the equivalence under rotation and reversal, as well as the isolated points $\left[U_{n}: L_{n}\right],\left[L_{n}: U_{n}\right]$.

Definition 4 Let $\gamma(B)$ be the graph with vertices $M=[A: B] \in \mathcal{M}_{n}$ and edges connecting $M, \tau(M)$.

As stated in Theorem 6 below, the graph $\gamma(B)$ is connected for any $B \in \mathcal{B}_{n}$. The proof is by induction, and follows from Theorems 4 and 5 and related definitions.

Observe that there are at least two $(i, j)$ with $|j-i|=1(\bmod 2 n)$ in any noncrossing perfect matching, and the two connecting arcs on the other side of such a "bump" in a meander are necessarily unobstructed.

Definition 5 Let $\beta_{t}$ be the arc $(t, t+1)$ for $1 \leq t<2 n$ and $\beta_{2 n}=(1,2 n)$.

Theorem 4 For $M=[A: B] \in \mathcal{M}_{n}$ with $\beta_{t} \in B, \beta_{t-1}(\bmod 2 n) \notin A$, there exists a meandric move $\tau(M)=\left[A^{\prime}: B\right]$ such that $\beta_{t-1(\bmod 2 n)} \in A^{\prime}$.

Proof: Assume without loss of generality that $(2 n-1,2 n) \in B,(2 n-2,2 n-1) \notin A$. We claim there is a meandric move $\tau(M)=\left[A^{\prime}: B\right]$ such that $(2 n-2,2 n-1) \in A^{\prime}$. The arcs

$$
(i, 2 n),(p, 2 n-1),(k, 2 n-2) \in A \text { with } 1 \leq i<p<k<2 n-2<2 n-1<2 n
$$

are a meandric triple with

$$
i \rightarrow 2 n \rightarrow 2 n-1 \rightarrow p \ldots k \rightarrow 2 n-2 \ldots
$$

A meandric move on these arcs yields $(i, p),(k, 2 n),(2 n-1,2 n) \in A^{\prime}=\sigma(\sigma(A))$.

There is an immediate dual result for $\beta_{t+1}(\bmod 2 n) \notin A$ under reversals. Next, Definition 6 makes precise the intuitive notion of contracting a bump $\beta_{2 n}$ from $A$ and the two connecting arcs in $B$ to produce a reduced meander of order $n-1$. In other words, the meander with arcs

$$
i \neg 1 \rightarrow 2 n \rightarrow j \rightarrow \overbrace{\cdots}^{R} \rightarrow i \text { reduces to } i \rightarrow j \rightarrow \overbrace{\cdots}^{S} \rightarrow i
$$

where the remaining arcs in $R$ stay the same in $S$ except for relabeling the endpoints to account for the removal of the $\operatorname{arc}(1,2 n)$ from $A$ and the replacement of $\operatorname{arcs}(1, i),(j, 2 n)$ in $B$ with $(i, j)$. 
Definition 6 For $M=[A: B] \in \mathcal{M}_{n}$ and $\beta_{2 n} \in A$, define $\rho(M, 2 n)$ to be $\left[A^{\prime}: B^{\prime}\right] \in \mathcal{M}_{n-1}$ with

$$
(i, j) \in B^{\prime} \quad \text { for } \quad(1, i),(j, 2 n) \in B \quad \text { and } \quad 1<i<j<2 n
$$

and, for $X=A, B$, with

$$
(k, l) \in X^{\prime} \quad \text { for } \quad(k+1, l+1) \in X \quad \text { and } \quad 1<k<l<2 n .
$$

For $\beta_{t} \in A$ with $1 \leq t<2 n$, the definition of $\rho(M, t)$ is fundamentally the same, although a precise statement of the replacement and relabeling is more complicated. If $\beta_{t} \notin A$, then $\rho(M, t)$ is not defined.

Theorem 5 The operation $\rho: \mathcal{M}_{n} \times\{1,2, \ldots, 2 n\} \rightarrow \mathcal{M}_{n-1}$ is well-defined.

Proof: Suppose without loss of generality that $(1,2 n) \in A$. The arcs of $A$ and $B$ form the meander $M$ :

$$
i \rightarrow 1 \rightarrow 2 n \rightarrow j \rightarrow \overbrace{\cdot}^{R} \rightarrow i
$$

Consider the exchange $\sigma_{P}(B)$ on $P=\{(1, i),(j, 2 n)\}$. Then $\left(A: \sigma_{P}(B)\right)$ has two closed loops:

$$
1 \rightarrow 2 n \rightarrow 1 \text { and } i \rightarrow j \rightarrow \overbrace{\cdots}^{R} \rightarrow i
$$

Under the appropriate endpoint relabeling, the second closed loop is the meander $\rho(M, 2 n)$.

Theorem 6 For $B \in \mathcal{B}_{n}$, the graph $\gamma(B)$ is connected.

Proof: Consider $M=[A: B]$ and $N=[C: B]$ for $B \in \mathcal{B}_{n}$ with $n>3$. Let $\beta_{t} \in B$. Suppose that $\beta_{s} \notin A \cup C$ for $s=t-1(\bmod 2 n)$. By Theorem 4 , there exist meandric moves $\tau(M)=\left[A^{\prime}: B\right]=M^{\prime}$ and $\tau(N)=\left[C^{\prime}: B\right]=N^{\prime}$ such that $\beta_{s} \in A^{\prime} \cap C^{\prime}$.

Observe that $\beta_{s}$ obstructs no arcs in either $A^{\prime}$ or $C^{\prime}$. By induction, $\rho\left(M^{\prime}, s\right)$ and $\rho\left(N^{\prime}, s\right)$ are connected by a sequence of meandric moves. Hence, there exists a sequence of meandric moves on the $n-1$ upper arcs of $M^{\prime}$ leaving the arc $\beta_{s} \in A^{\prime} \cap C^{\prime}$ fixed and connecting $M^{\prime}$ to $N^{\prime}$ in $\gamma(B)$.

\section{Some characteristics of meander graphs}

The proof of Theorem 6 implies that the diameter of $\gamma(B)$ is at most $2 n$ for $B \in \mathcal{B}_{n}$. This upper bound is never achieved since for $3 \leq n \leq 8$, the maximum diameter of $\gamma(B)$ is $n-2$.

Example 3 When $n=9$, there is one (nonisomorphic) pair of meanders $[A: B]$ and $\left[A^{\prime}: B\right]$ whose geodesic has 8 meandric moves in $\gamma(B)$ :

$$
\begin{aligned}
& B=\{(1,10),(2,9),(3,8),(4,5),(6,7),(11,18),(12,17),(13,14),(15,16)\} \\
& A=\{(1,16),(2,15),(3,14),(4,13),(5,12),(6,11),(7,10),(8,9),(17,18)\} \\
& A^{\prime}=\{(1,4),(2,3),(5,18),(6,17),(7,16),(8,15),(9,14),(10,13),(11,12) .\}
\end{aligned}
$$



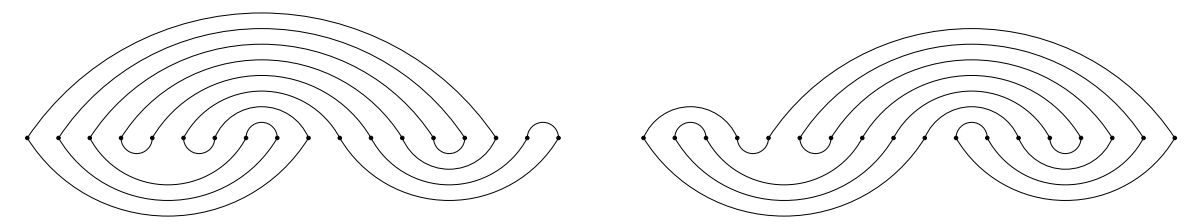

Fig. 4: The smallest instance of meanders $[A: B]$ and $\left[A^{\prime}: B\right]$ with geodesic length $\neq n-2$ from Example 3
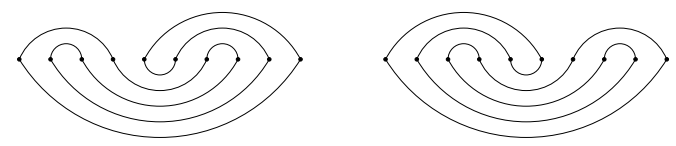

Fig. 5: The smallest instance of interlocking meanders $[A: B]$ and $\left[A^{\prime}: B\right]$ from Example 4

This is the only pair of meanders, up to rotation and reversal, whose geodesic has length greater than $n-2$ for $n=9$. When $n=10$, there are three nonisomorphic pairs with length 9 .

We contrast this with $\mathcal{G}_{n}$ which inherits a diameter of $n-1$ from $\mathrm{NC}(2 n$, match) under matching exchange. If $\beta_{t} \notin A$, then there is always an exchange such that $\beta_{t} \in \sigma(A)$. This is not the case for meandric moves; the smallest example is the following.

Example 4 When $n=5$, there is one (nonisomorphic) pair of meanders $M=[A: B]$ and $M^{\prime}=\left[A^{\prime}: B\right]$ such that for every $\beta_{t} \in A$ there exists no $\tau\left(M^{\prime}\right)=\left[A^{\prime \prime}: B\right]$ with $\beta_{t} \in A^{\prime \prime}$, and vice versa:

$$
\begin{aligned}
& B=\{(1,10),(2,9),(3,8),(4,7),(5,6)\} \\
& A=\{(1,4),(2,3),(5,10),(6,9),(7,8)\} \\
& A^{\prime}=\{(1,6),(2,5),(3,4),(7,10),(8,9) .\}
\end{aligned}
$$

We say that such a pair of meanders is interlocking. There are no interlocking pairs when $n=6$, eight when $n=7$, seven when $n=8$, and 198 when $n=9$.

Yet, any interlocking pair is still connected in $\gamma(B)$. Hence, for $\beta_{t} \in A^{\prime}$ and $\beta_{t} \notin A$, there is always a sequence of meandric moves $\tau(\ldots \tau(M))=\left[A^{*}: B\right]$ such that $(t, t+1) \in A^{*}$.

Theorem 7 Let $B \in \mathcal{B}_{n}$ and $\beta_{t} \notin B$. Then there exists $M=[A: B]$ such that $\beta_{t} \in A$.

Proof: The proof essentially inverts the map $\rho$ in Definition 6. Assume $t=2 n$. Let $B^{\prime}$ be the arcs with

$$
\begin{aligned}
& (i, j) \in B^{\prime} \quad \text { for } \quad(1, i),(j, 2 n) \in B \quad \text { and } \quad 1<i<j<2 n \\
& (k, l) \in B^{\prime} \quad \text { for } \quad(k+1, l+1) \in B \quad \text { and } \quad 1<k<l<2 n \text {. }
\end{aligned}
$$

Then $B^{\prime} \in \mathcal{B}_{n-1}$ and there exists $A^{\prime} \in \mathcal{A}_{n-1}$ such that $\left[A^{\prime}: B^{\prime}\right] \in \mathcal{M}_{n-1}$. Let $A$ be the set of arcs with

$$
(k, l) \in A \quad \text { for } \quad(k-1, l-1) \in A^{\prime} \quad \text { and } \quad 1<k<l<2 n .
$$

Then by construction $[A: B] \in \mathcal{M}_{n}$.

Consequently, for every $\beta_{t} \notin B$, there is a subgraph of $\gamma(B)$ isomorphic to $\gamma\left(B^{\prime}\right)$ as in the proof of Theorem 7. By the proof of Theorem 4, every $M \in \gamma(B)$ is at most distance one from the subgraphs containing $\rho(M, t-1(\bmod 2 n))$ and $\rho(M, t+1(\bmod 2 n))$ for each $\beta_{t} \in B$. 
We also have the following result. Although it is an immediate corollary to Theorems 6 and 7 , we give here a constructive proof to illustrate some of the challenges in working with meandric triples.

Theorem 8 Let $M=[A: B] \in \mathcal{M}_{n}$. For $\beta_{t} \notin B$, there exists a sequence of meandric moves $\tau(\ldots \tau(M))=\left[A^{*}: B\right]$ such that $\beta_{t} \in A^{*}$.

Proof: Assume $t$ is odd. For $k, l \neq t+1$ and $k^{\prime}, l^{\prime} \neq t$, the meander $M$ has arcs

$$
k \rightarrow t \rightarrow \overbrace{l \ldots l^{\prime}}^{R} \rightarrow t+1 \rightarrow \overbrace{k^{\prime} \ldots k}^{R^{\prime}} .
$$

Since $\beta_{t} \notin B$, there is at least one arc from $A$ in the sequence of $\operatorname{arcs} R^{\prime}$. Suppose there exists $i \rightarrow j$ in $R^{\prime}$ which forms a meandric triple with $t \rightarrow l$, and $l^{\prime} \rightarrow t+1$. Then $(t, t+1) \in \tau(M)$.

If not, then consider $i \rightarrow j$ from $R^{\prime}$ having $d$ arcs from $R$ which obstruct it from forming a meandric triple with $t \rightarrow l, l^{\prime} \rightarrow t+1$. If $d>2$, then a meandric move on three of the $d$ arcs, which must be a meandric triple, yields $\tau(M)$ which now has $d-2$ obstructing arcs. Hence, the relevant cases are when there are 1 or 2 obstructing arcs.

The three cases for a linear ordering of the points from $t \rightarrow l$ and $l^{\prime} \rightarrow t+1$ are equivalent under rotations and reversals. Suppose that $t<t+1<l^{\prime}<l$. The endpoints $1, \ldots, 2 n$ are divided into three sets by the two arcs: $S_{1}=\{i \mid 1 \leq i<t, l<i \leq 2 n\}, S_{2}=\left\{i \mid t+1<i<l^{\prime}\right\}$, and $S_{3}=\left\{i \mid l^{\prime}<i<l\right\}$. Then $i, j$ and the endpoints of the obstructing arcs must all be in one of the three sets. Moreover, the case when they lie in $S_{1}$ is equivalent to $S_{3}$.

Suppose there is a single obstructing arc $a \rightarrow b$ :

$$
t \rightarrow l \overbrace{\ldots a \rightarrow b \ldots .}^{R} l^{\prime} \rightarrow t+1 \overbrace{\ldots i \rightarrow j \ldots .}^{R^{\prime}}
$$

We explicitly consider the two situations when either

$$
a<j<i<b<t<t+1<l^{\prime}<l \text { or } t<t+1<l^{\prime}<b<i<j<a<l .
$$

In the second case when the arcs lie in $S_{2}$, operating on $M$ by a meandric move on $i \rightarrow j, a \rightarrow b$, and $t \rightarrow l$ followed by a move on the new meandric triple $i \rightarrow l, t \rightarrow b, l^{\prime} \rightarrow t+1$ results in $\beta_{t} \in \tau(\tau(M))$. We claim the first case, when the arcs lie in $S_{1}$, results in a contradiction.

Consider $n=4$. Then the closed loop would be

$$
t \rightarrow l \rightarrow a \rightarrow b \rightarrow l^{\prime} \rightarrow t+1 \rightarrow i \rightarrow j \rightarrow t .
$$

However, it is not possible to have the three arcs $t+1 \rightarrow i, j \rightarrow t$ and $b \rightarrow l^{\prime}$ lying below the horizontal line without intersections. Suppose $n>4$ and there is a meander $M \in \mathcal{M}_{n}$ containing the arrangement of four arcs. There exists an additional arc $i^{\prime \prime} \rightarrow j^{\prime \prime}$ where $\left|i^{\prime \prime}-j^{\prime \prime}\right|=1$. Without loss of generality, $j^{\prime \prime}=i^{\prime \prime}+1$ and $\rho\left(M, i^{\prime \prime}\right)$ has $n-1$ arcs. Inductively, though, the arcs in $\rho\left(M, i^{\prime \prime}\right)$ corresponding to $t+1 \rightarrow i, j \rightarrow t$ and $b \rightarrow l^{\prime}$ intersect.

Suppose now that there are two obstructing arcs $a \rightarrow b, a^{\prime} \rightarrow b^{\prime}$ between $i \rightarrow j$ and $t \rightarrow l, l^{\prime} \rightarrow t+1$. There are two distinct orderings for $a, b$ and $a^{\prime}, b^{\prime}$ along the horizontal line with respect to the other arcs. When the obstructing arcs lie in $S_{1}$, one ordering results in a contradiction like the one above while the other yields $\beta_{t} \in \tau(\tau(M))$. When the obstructing arcs lie in $S_{2}$, then both orderings result in a contradiction. 


\section{Concluding remarks}

The present work raises several interesting directions for further study. For instance, it would be very helpful to have an appropriate statistic to measure how close a given meander is to a random one. Such a statistic would offer ways to measure the "autocorrelation time" of the Markov chain as well as help in bounding its approach to equilibrium. Simulating the Markov chains proposed here and observing "random" meanders after a large number of steps might be one way to come up with some interesting statistics on meanders. Given the simplicity of the chains, this should be a relatively straight-forward task, which we hope to undertake in the near future.

There are also unanswered questions about the structure of meander graphs $\mathcal{G}_{n}$ and $\gamma(B)$ for $B \in \mathcal{B}_{n}$. For instance, we give a tight upper bound of $\mathcal{O}\left(n^{4}\right)$ on the maximum vertex degree in $\mathcal{G}_{n}$, but have not yet fully investigated the amount of variation in vertex degrees over the graph. Similarly, our bounds on the diameter and maximum vertex degree hold for all $\gamma(B)$ over $B \in \mathcal{B}_{n}$. Yet, it is clear from the graphs for small $n$ that these characteristics depend in some unknown way on the particular $B$.

Finally, this MCMC approach to counting and sampling closed meanders extends to other types as well. In particular, semi-meanders of order $n$ are in bijection with the subset of closed meanders of order $n$ with the "rainbow" matching on the bottom: $[A: R]$ where $R=\{(i, 2 n-i+1) \mid 1 \leq i \leq n\}$. Likewise, there is a correspondence between closed meanders and open meanders of odd order, and a many-to-one mapping from closed meanders to open meander of even order.

\section{Acknowledgments}

The authors thank the anonymous reviewers whose comments significantly improved the paper, and Geehoon Hong for improving the naive degree bound on $\gamma(B)$.

This research is supported in part by NSF DMS-0701043 to Prasad Tetali, and by a Career Award at the Scientific Interface (CASI) from the Burroughs Wellcome Fund (BWF) to Christine Heitsch.

\section{References}

[AK80] Jaromír Abrham and Anton Kotzig. Transformations of Euler tours. Ann. Discrete Math., 8:65-69, 1980. Combinatorics 79 (Proc. Colloq., Univ. Montréal, Montreal, Que., 1979), Part I.

[AP05] M. H. Albert and M. S. Paterson. Bounds for the growth rate of meander numbers. J. Combin. Theory Ser. A, 112(2):250-262, 2005.

[CJ03] S. Cautis and D. M. Jackson. The matrix of chromatic joins and the Temperley-Lieb algebra. J. Combin. Theory Ser. B, 89(1):109-155, 2003.

[DF00a] P. Di Francesco. Exact asymptotics of meander numbers. In Formal power series and algebraic combinatorics (Moscow, 2000), pages 3-14. Springer, Berlin, 2000.

[DF00b] P. Di Francesco. Folding and coloring problems in mathematics and physics. Bull. Amer. Math. Soc. (N.S.), 37(3):251-307 (electronic), 2000.

[DFGG00] P. Di Francesco, O. Golinelli, and E. Guitter. Meanders: exact asymptotics. Nuclear Phys. B, 570(3):699-712, 2000. 
[DS98] Persi Diaconis and Bernd Sturmfels. Algebraic algorithms for sampling from conditional distributions. Ann. Statist., 26(1):363-397, 1998.

[FE02] Reinhard O. W. Franz and Berton A. Earnshaw. A constructive enumeration of meanders. Ann. Comb., 6(1):7-17, 2002.

[Fra98] Reinhard O. W. Franz. A partial order for the set of meanders. Ann. Comb., 2(1):7-18, 1998.

[Gol00] O. Golinelli. A Monte-Carlo study of meanders. Eur Phys J B, 14(1):145-155, 2000.

[Hal06] H. Tracy Hall. Meanders in a cayley graph, 2006.

[Hei] Christine E. Heitsch. A new metric on plane trees and RNA configurations. In revision.

[Jen00] Iwan Jensen. A transfer matrix approach to the enumeration of plane meanders. J. Phys. A, 33(34):5953-5963, 2000.

[Jer03] Mark Jerrum. Counting, sampling and integrating: algorithms and complexity. Lectures in Mathematics ETH Zürich. Birkhäuser Verlag, Basel, 2003.

[JM96] Mark T. Jacobson and Peter Matthews. Generating uniformly distributed random Latin squares. J. Combin. Des., 4(6):405-437, 1996.

[Kre72] G. Kreweras. Sur les partitions non croisées d'un cycle. Discrete Math., 1(4):333-350, 1972.

[KS91] Ki Hyoung Ko and Lawrence Smolinsky. A combinatorial matrix in 3-manifold theory. Pacific J. Math., 149(2):319-336, 1991.

[Lun68] W. F. Lunnon. A map-folding problem. Math. Comp., 22:193-199, 1968.

[LZ93] S. K. Lando and A. K. Zvonkin. Plane and projective meanders. Theoret. Comput. Sci., 117(1-2):227-241, 1993. Conference on Formal Power Series and Algebraic Combinatorics (Bordeaux, 1991).

[MT99] Lisa McShine and Prasad Tetali. On the mixing time of the triangulation walk and other Catalan structures. In Randomization methods in algorithm design (Princeton, NJ, 1997), volume 43 of DIMACS Ser. Discrete Math. Theoret. Comput. Sci., pages 147-160. Amer. Math. Soc., Providence, RI, 1999.

[MT06] Ravi Montenegro and Prasad Tetali. Mathematical aspects of mixing times in Markov chains. Found. Trends Theor. Comput. Sci., 1(3):x+121, 2006.

[Sav09] David Savitt. Polynomials, meanders, and paths in the lattice of noncrossing partitions. Trans. Amer. Math. Soc., 361(6):3083-3107, 2009.

[Sim00] Rodica Simion. Noncrossing partitions. Discrete Math., 217(1-3):367-409, 2000. Formal power series and algebraic combinatorics (Vienna, 1997).

[Tou50] Jacques Touchard. Contribution à l'étude du problème des timbres poste. Canadian J. Math., 2:385-398, 1950. 